\title{
Phylogenetic Analysis of Trypanosoma evansi Isolates in Naturally Infected Camels from Kingdom of Saudi Arabia
}

\author{
N. I. Elobaid ${ }^{*}$, O. M. Daffalla ${ }^{2}$, E. M. Noureldin ${ }^{2}$ and M. A. Abdalla ${ }^{1}$ \\ ${ }^{1}$ Department of Preventive Medicine and Public Health, College of Veterinary Medicine, \\ Sudan University of science and Technology \\ ${ }^{2}$ Saudi National Centre for Disease Prevention and Control (SCDC) - Jazan, Saudi Arabia \\ *Corresponding author
}

Keywords

Mortality, ribosomal DNA cistron genes, ribosomal RNA, fertility

Article Info

\section{Accepted:}

18 March 2021 Available Online: 10 April 2021
Internal Transcribed Spacer1 (ITS-1) of nuclear ribosomal DNA (rDNA) sequences of Trypanosoma evansi isolates from camel dromedary of King of Saudi Arabia (KSA) used to investigate evolutionary origins and infer phylogenetic relationships with other Global isolates. The study was actually started with microscopic surveillance of blood samples on 454 from naturally infected camels in Jazan region, south western, by using wet and thick smear film which revealed 3.1\% (14 positive) and 3.5\% (16 positive), respectively, however molecular examination was performed on 100 blood samples using conventional PCR- ITS1 targeting $480 \mathrm{bp}$ and resulted 19\% positive, the ITS1 product was subjected to RoTat 1.2 VSG primer $(151 \mathrm{bp})$ that derived from a trypanosome specific repetitive nucleotide sequence fragment and yielded $36.8 \%$ positive. The molecular characterization and phylogenetic analysis were achieved on product sequences and aligned against the corresponding GenBank sequences of known isolates of T. evansi. Basic Local Alignment Search Tool (BLAST) data of the obtained ITS1 gene sequences showed identity to those of $T$. evansi, with the homology of $92 \%$ to $99 \%$. Sequence analysis of this gene generated six heterogeneous genotypes of $T$. evansi in KSA are present, released in GenBank and encoded in following terms of jaz1, jaz2, jaz3, jaz4, jaz5 and jaz6 with accession numbers; MN611173, MN625863, MN625864, MN611174, MN625865 and MN625866, respectively. T. evansi isolates from KSA and from other countries were tightly clustered into the constructed phylogenetic tree based on the ITS-1 rDNA sequence using Maximum Composite Likelihood and neighbour-joining method, revealing apparently genetic diversity among the isolates. This isthe first study about sequence analysis of T. evansi which prove genetic diversity within isolates from Saudi Arabia.

\section{Introduction}

Trypanosomosis is considered to be one of the most important vector-borne and a ubiquitous disease represents a major threat to domestic and non-domestic animals, caused by a flagellated protozoan parasite that is Trypanosoma evansi. Camel trypanosomosis 
"Surra", or "Heyam" as known in Saudi Arabia, was first reported in the kingdom by Kasim(1). More recently, studies were conducted in Jazan region to estimate the prevalence of the disease $(2,3)$. Trypanosomosis imposes significant financial losses due to mortality and reduced fertility (4), thus, it has several clinical forms as acute, sub-acute, chronic and in apparent (5). Trypanosomes have two genomes, one within the nucleus and the other enclosed within the kinetoplast (mitochondrial DNA) (6). Nuclear DNA bears genes coding for ribosomal RNA and ribosomal DNA cistron genes which occur in multiple copies in cycle arrays (7), whereby indicated these genes are made of transcriptional units (TU), separated by nontranscribed spacers (NTS). The TU is composed of an $18 \mathrm{~S}$ ribosomal subunit, internal transcribed spacer 1 (ITS-1), 5.8S ribosomal subunit, ITS-2 and $28 \mathrm{~S}$ ribosomal subunit. The length of ITS-1 is about 300-800 base pairs (bp) and has a variable length size depending on the Kinetoplastida species (7). The ITS1 and ITS2 genes can be successfully used to differentiate different species in the genus Trypanosoma either using ITS or KIN primers, additionally, 18S rRNA and glyceraldehyde 3-phosphate dehydrogenase genes (gGAPDH) can be used to confirm monophyly in the trypanosome evolution. Whereas the mitochondrial DNA is a needful structure of Kinetoplastida spp. that are distinguished by the presence of a DNA (kDNA) containing region, known as a kinetoplast in their single large mitochondrion (8). The kinetoplast contains circular DNA in two forms, maxicircles and minicircles. However T. evansi are dyskinetoplastic (kDNA-) since they lack part of the kDNA $(9,10)$. Molecular analysis of the genomic or mitochondrial DNA so as Restriction Fragment Length Polymorphisms (RFLPs) and PCR-Random Amplified polymorphic DNA (PCR-RAPD), or the utilization of microsatellite and minisatellite markers have been used successfully for identification of Phylogenetics of trypanosome species (11). Other different molecular techniques were also used to provide information on the $T$. evansi population genetic structure and the genetic diversity using published sequences from different countries in Africa, Asia and Latin America (12-14). Some specific genes have been sequenced, aligned and analysed in order to study the phylogenetic relationships among morphologically indistinguishable trypanosomes (15), as an example, ITS-1 and ITS-2 of the rDNA, which are useful targets for species delineation and for inferring phylogenetic relationships of Trypanosoma spp. $(12,16)$. Evolutionary events also have been addressed by studying the ribosomal RNA (rRNA) genes and their associated spacer regions, collectively called ribosomal DNA (17). Wherefore, this study is planned to provide data on disease prevalence using Nested-PCR amplification based on Internal Transcribed Spacer 1 (ITS-1) and TeRoTat primers, and to investigate genetic characterization and phylogenesis of $T$. evansi isolates from naturally infected camels in Jazan region, south western Saudi Arabia.

\section{Materials and Methods}

\section{Study area}

The present study was conducted in 9 governorates of Jazan region (Jazan, Samttah, Abuareesh, Sabyah, Beash, Al-Darb, AlAridah, Al-Ahad and Al-Khobah). Jazan region, occupies an area of approximately $40,457 \mathrm{~km}$, is located in the southwest of Saudi Arabia between $16^{\circ}-12$, and $18^{\circ}-25$, latitude north. Bordered on the north and east by the Asir region and the Red Sea on the west along the coastline about $260 \mathrm{~km}$, whereas the Republic of Yemen from the south and southeast. The human population is estimated at about 1,603,659 (18). The region includes more than 3500 villages and over 100 islands 
located in the Red Sea. (Fig.1), the geographic features are arranged in deserts, mountain ranges and open-low plains (less than 400 meters above sea level). It is drained by several permanent valleys which play a crucial role in providing the perennial breeding habitats for insects, and several intermittent valleys (19). The Jazan region is situated in the subtropical zone and has average monthly temperatures ranging from $25.8^{\circ} \mathrm{C}$ in January to $33.4^{\circ} \mathrm{C}$ in July. The average relative humidity ranges between $55 \%$ and $72.5 \%$ (20).

Fig.1 Map of Jazan region showing different governorates.

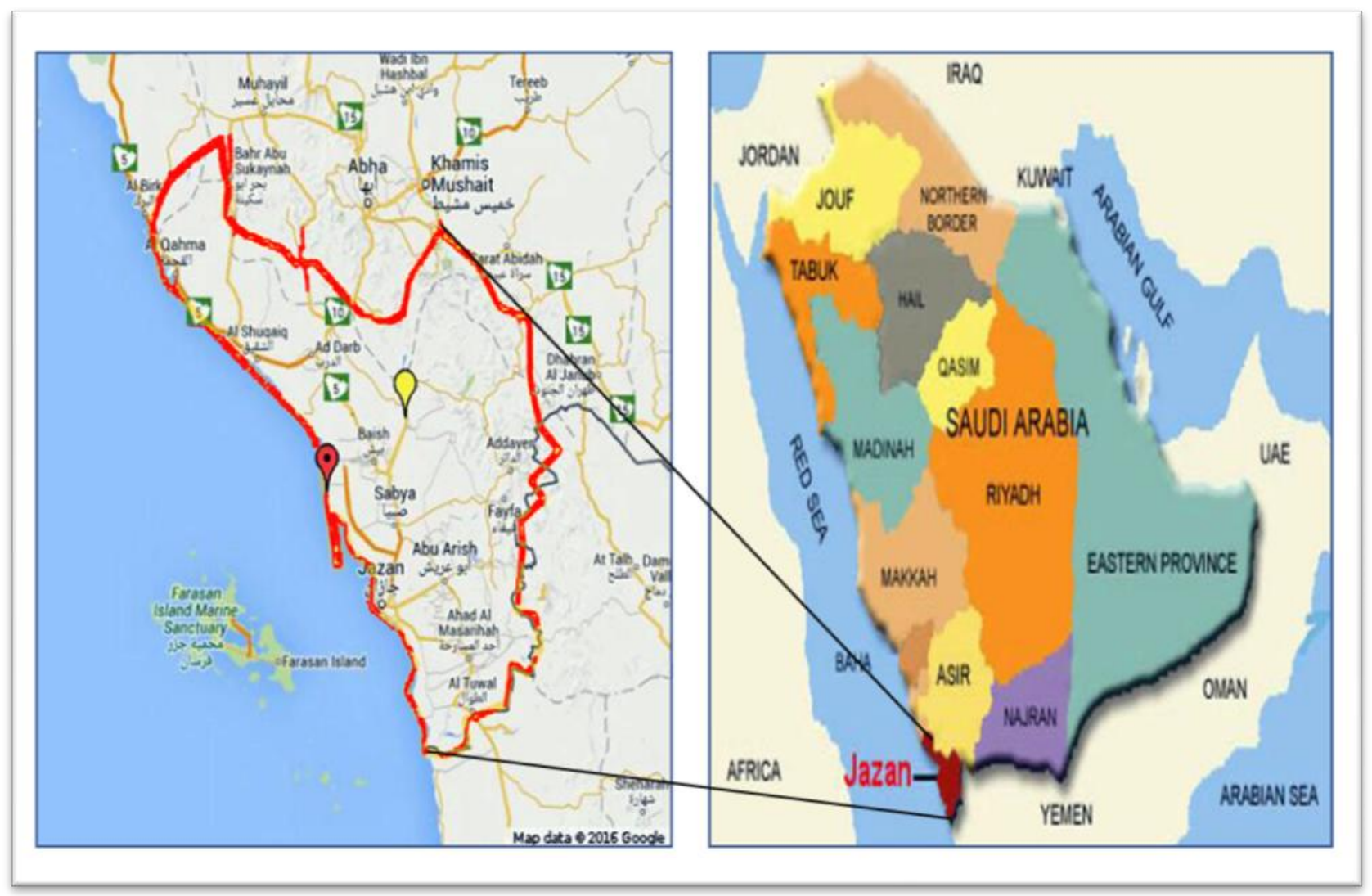

Annual rainfalls vary according to the area, in the plains, it ranges between $200-500 \mathrm{~m}$ min August and falling again in the months of March and April, while inmountains average from 529to630mm (www.jazan.gov.sa). The vicinity of the region is considered to be a border point to the Yemen state and African Horne, in addition to the seaport, which plays a vital role in livestock's movement in and out of the country, and hence, provides the possibility of transmission of many infectious diseases.

\section{Sample collection}

During the period of December 2017 to April 2018, a total of 454 blood samples were collected from camels' herds in Jazan region. Blood samples were collected from camel by jugular vein puncture using EDTA-coated vacutainer tubes. All blood samples were labelled accordingly, tested by microscopic examinations at the field and then packed in a cooler bag with ice packs before they were couriered to the laboratory for DNA extraction and PCR analysis. 


\section{Wet Blood Film}

One drop of blood was placed on a clean slide, covered with a cover slip to spread the blood as a monolayer of cells, scanned under a light microscope using $\times 10$ and changed to the $\times 40$ objective lens to detect any motile trypanosome (21).

\section{Stained thick smear film}

A drop of blood was placed on the centre of microscope slide, spread, placed and stained with Giemsa then picked off singly, the stain was flushed off with purified water, and left the slide to dry to examine under light microscope using oil immersion with $\times 100$ objective lens.

\section{Extraction of genomic DNA and purification}

DNA was extracted from stored and confirmed microscopic samples of camel blood using Wizard genomic DNA Extraction kit (Promega, U.S.A) following the manufacturer's recommendations: $300 \mu 1$ of blood added to $900 \mu \mathrm{l}$ of cell lysis solution in 1.5 tube and incubate the mixture for ten minutes at room temperature (invert 2-3 times once during the incubation).

Then centrifuged at $13000 \mathrm{rpm}$ for 20 seconds at room temperature then the supernatant discarded and the pellet was resuspended by vortex for 15 seconds and $300 \mu \mathrm{l}$ of Nuclei Lysis Solution added to resuspended cells, then $100 \mu \mathrm{l}$ of Protein Precipitation was added.

The mixture was purified by centrifugation at $13000 \mathrm{rpm}$ for 3 minutes and the supernatant transferred to a clean $1.5 \mathrm{ml}$ tube containing $300 \mu \mathrm{l}$ of isopropanol and mixed gently. The DNA was pelleted at $13000 \mathrm{rpm}$ for 1 minute and washed by ethanol twice and dried for 5 -
10 minutes at room temperature. DNA Rehydration solution used to re-suspend the DNA at $65^{\circ} \mathrm{C}$ for 1 hour. The extracted DNA stored at $-86^{\circ} \mathrm{C}$ until $\mathrm{PCR}$ processing could be completed.

\section{PCR amplification protocol}

PCR was carried out for detection and identification of Trypanosoma species as described by Njiru(22) and Salim (13). DNA samples were amplified by oligonucleotide primers obtained from Integrated DNA technology (Belgium).

In primary amplification, the sample subject to PCR using ITS1 CF/BR FP and ITS1 CF/BR RP (22) primer which amplifies 480 bp of ITS1 region of the rDNA gene of all Trypanozoon. Further, positive ITS1 Trypanosoma species were also submitted to PCR test using TeRoTat920F and TeRoTat1070R. This primer specific for $T$. evansi which a set that amplifies $151 \mathrm{bp}$ of the T. evansiRoTat 1.2 VSG gene fragment was used (23), Table.(1)showed primers were used). In brief PCR were carried out in total $25 \mu \mathrm{l}$ reaction volume, each containing $12.5 \mu \mathrm{l}$ GoTag®G2 green master mix ready to use from Promega and $25 \mu \mathrm{M}$ of each primer, $5 \mu 1$ of extracted DNA was used as sample for the primary amplification and $1 \mu \mathrm{l}$ of the PCR product. In each run, negative and positive controls were included. Thermal cycling was done in T100 thermal cycler (Bio-Rad, USA), PCR conditions programmed as follows: Initial denaturation at $95^{\circ} \mathrm{C}$ for $2 \mathrm{~min}, 35$ cycles of denaturation at $95^{\circ} \mathrm{C}$ for $30 \mathrm{sec}$, annealing at $58^{\circ} \mathrm{C}$ for $30 \mathrm{sec}$ and $55^{\circ} \mathrm{C}$ for 90 sec, extension at $72^{\circ} \mathrm{C}$ for $1 \mathrm{~min}$ and final extension step of $5 \mathrm{~min}$. The PCR products were analyzed by gel electrophoresis (2\% Agarose in Tris-Acetate EDTA buffer) staining with ethidium bromide. The visualization was carried out using Gel Doc XR Imaging System (Bio-Rad). 


\section{Sequencing of PCR Fragment}

Purification and standard sequencing for ITS1- PCR products were performed by Macrogen Company (Seoul, Korea). Sequencing reactions were performed in a MJ Research PTC-225 Peltier Thermal Cycler using a ABI PRISM $®$ BigDyeTM Terminator Cycle Sequencing Kits with AmpliTaq ${ }^{\circledR}$ DNA polymerase (FS enzyme) (Applied Biosystems), following the protocols supplied by the manufacturer. Single-pass sequencing was performed on each template using D1 (forward) primer.

The fluorescent-labeled fragments were purified from the unincorporated terminators with BigDye $\AA X$ Terminator ${ }^{\mathrm{TM}}$ purification protocol.

The samples were resuspended in distilled water and subjected to electrophoresis in an ABI 3730xl sequencer (Applied Biosystems).

\section{Bioinformatics and sequence analysis}

The sequences were searched for genetic similarity through BLAST data (www.ncbi.nlm.nih.gov/BLAST/) (24) and compared to reference sequences of Trypanosoma isolates detected in the BLAST and downloaded from GenBank (www.ncbi.nlm.nih.gov/genbank/).

\section{Phylogenetic analysis}

Multiple sequence alignment for ITS1- PCR products was performed using 'Blosum 62' table (25) and Neighbor-Joining phylogenetic tree of ITS1- PCR products nucleotide squences was run using neighbor-joining algorithm by Mega7 programme (http://www.megasoftware.net/) (26).

The evolutionary distances were computed using the Maximum Composite Likelihood method (27) and are in the units of the number of base substitutions per site, the evolutionary history was inferred using the NeighborJoining method (28).

\section{Results and Discussion}

\section{Clinical signs}

During the investigation of camel trypanosomosis, it was observed that some of the examined camels showed clinical signs of dullness, loss of appetite, eye lacrimation, reduced milk yield and increase of body temperature.

\section{Microscopic examination of blood samples and PCR}

The prevalence of $T$. evansi infection in camels was found to be as follows: Out of 454 tested camels; $14(3.1 \%)$ and $16(3.5 \%)$, were found positive using wet and thick smear film respectively (Table 2). Among 100 camels examined by PCR-ITS1 (480 bp) were found 19 (19\%) positive. (Fig.2), then subjected to Rotat VSG 1.2- PCR primer and yielded seven positive (36.8\%) with $151 \mathrm{bp}$ band size (Fig.3).

\section{Bioinformatics and sequence analysis}

To confirm the results, a partial sequencing was done and amplified successfully for nineteen ITS1-PCR product samples representing the Trypanosoma species. The BLAST data searched for nucleotide sequences and generated six distinct genotypes of our samples isolates are present in Jazan region, they were encoded in following terms of jaz1, jaz2, jaz3, jaz4, jaz5 and jaz6 released in GenBank with accession numbers; MN611173, MN625863, MN625864, MN611174, MN625865 and MN625866, respectively. BLAST analysis resulted the sequences of KSA isolates of $T$. evansi had higher similarity with other known isolates of Iraq MH595480, Iran KX898420, 
Sudan LC492122, Egypt AB551922, Japan D89527, China FJ712715, Thailand AY912279, Netherland AF306775.1, Philippine HQ593646 and Indonesia MN121258, showed different identical from $99 \%$ to $92 \%$.

\section{Phylogenetic analysis}

The phylogenetic tree showed the evolutionary relationship of the sequences in which the length of the branch was proportional to the estimated genetic distance between the sequences (Fig.4). It exhibited various proportions of bootstrap values among clades that were unable to discriminate clearly the genetic diversity of Trypanosoma spp. isolates retrieved from GenBank. The multiple sequence alignment of ITS1 demonstrated sequence divergence of KSA isolates and other Known isolates from GenBank. Our isolates gave a high heterogeneity when sequences replicated 490 and showed genetic substitutions points and distances as follow; jaz1 $=16$, jaz2 $=20$, jaz3 $=38$, jaz $4=6$, jaz $5=59$ and jaz6=19. (Fig.5),

Many previous studies in Jazan region, south western Saudi Arabia, were undertaken to reveal the epidemiology of camel trypanosomosis, and thence recorded a high considerable rate in two occasions, Al-Khalifa (30), found $40 \%$ of infections in Jazan only out of six regions of the KSA, whilst, AlArabi (3), resulted of $22.2 \%$, has been represented a highest rate of the prevalence among the infected camels. Thus, whatever diagnostic method was applied, the current study showed a low proportion of prevalence of $T$. evansi infection when compared.

Fig.2 Agarose gel electrophoresis, stained with ethidium bromide of ITS1 CF/BR PCR, Lane (1) 100bp DNA marker. Products: lane (2) negative, lanes (3-7) positive Trypanozoon.

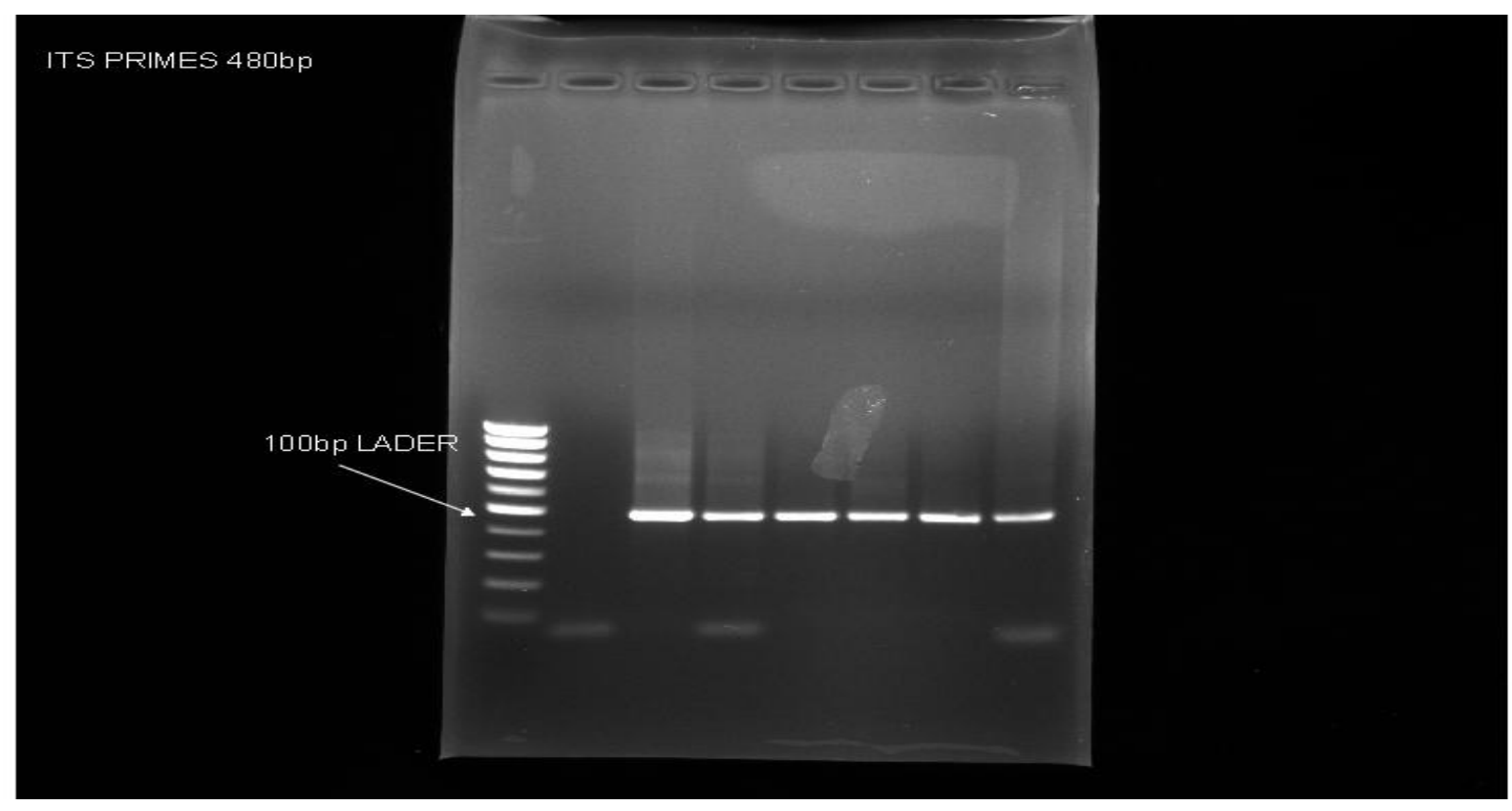


Fig.3 Agarose gel electrophoresis, stained with ethidium bromide of TeroRotat 1.2 PCR, Lane (1) 100bp DNA marker. Products: lanes (2-7) positive specific T. evansi.

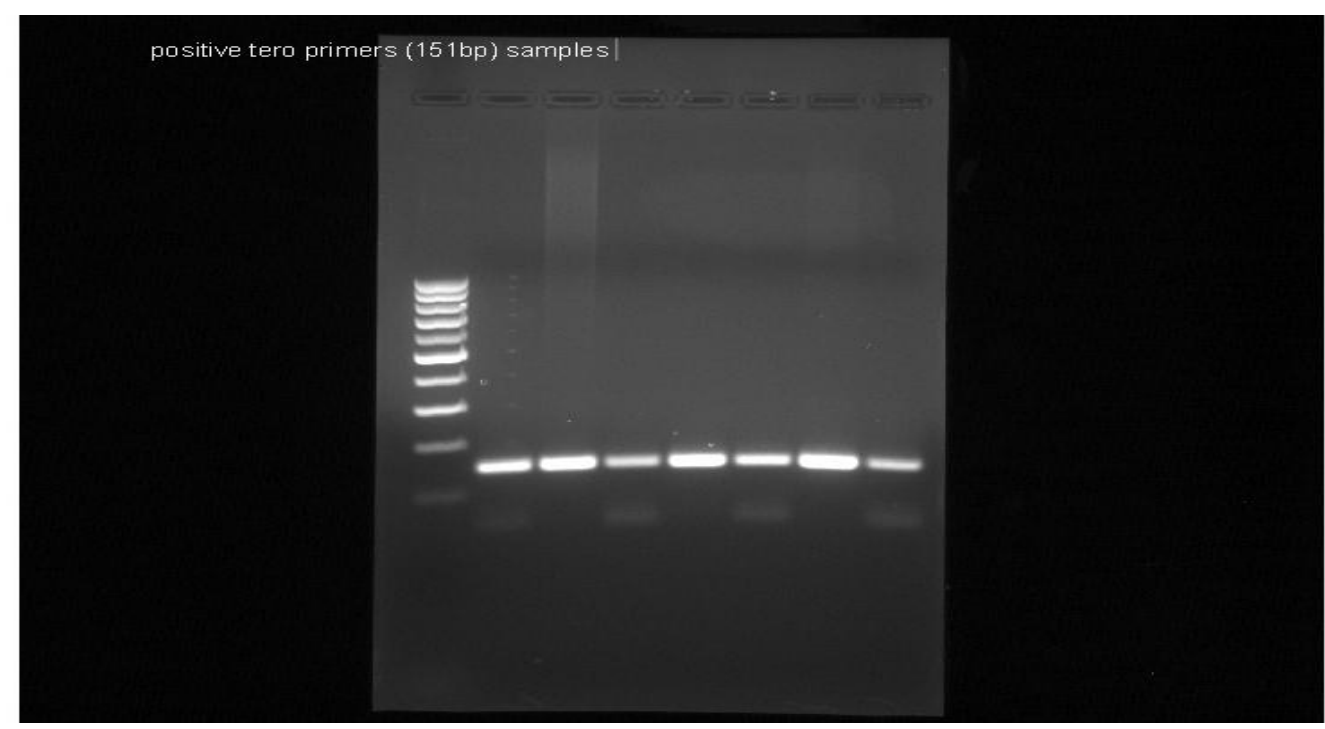

Fig.4 Phylogenetic tree based on ITS-1 sequences from trypanosomes of the Kingdom of Saudi Arabia with different countries' isolates. The optimal tree with the sum of branch length = 0.19072993 is shown, the percentage of replicate trees in which the associated taxa clustered together in the bootstrap test (500 replicates) is shown next to the branches (29). The tree is drawn to scale, with branch lengths in the same units as those of the evolutionary distances used to infer the phylogenetic tree, this analysis involved 16 nucleotide sequences and all ambiguous positions were removed for each sequence pair (pairwise deletion option), there were a total of 700 positions in the final dataset.

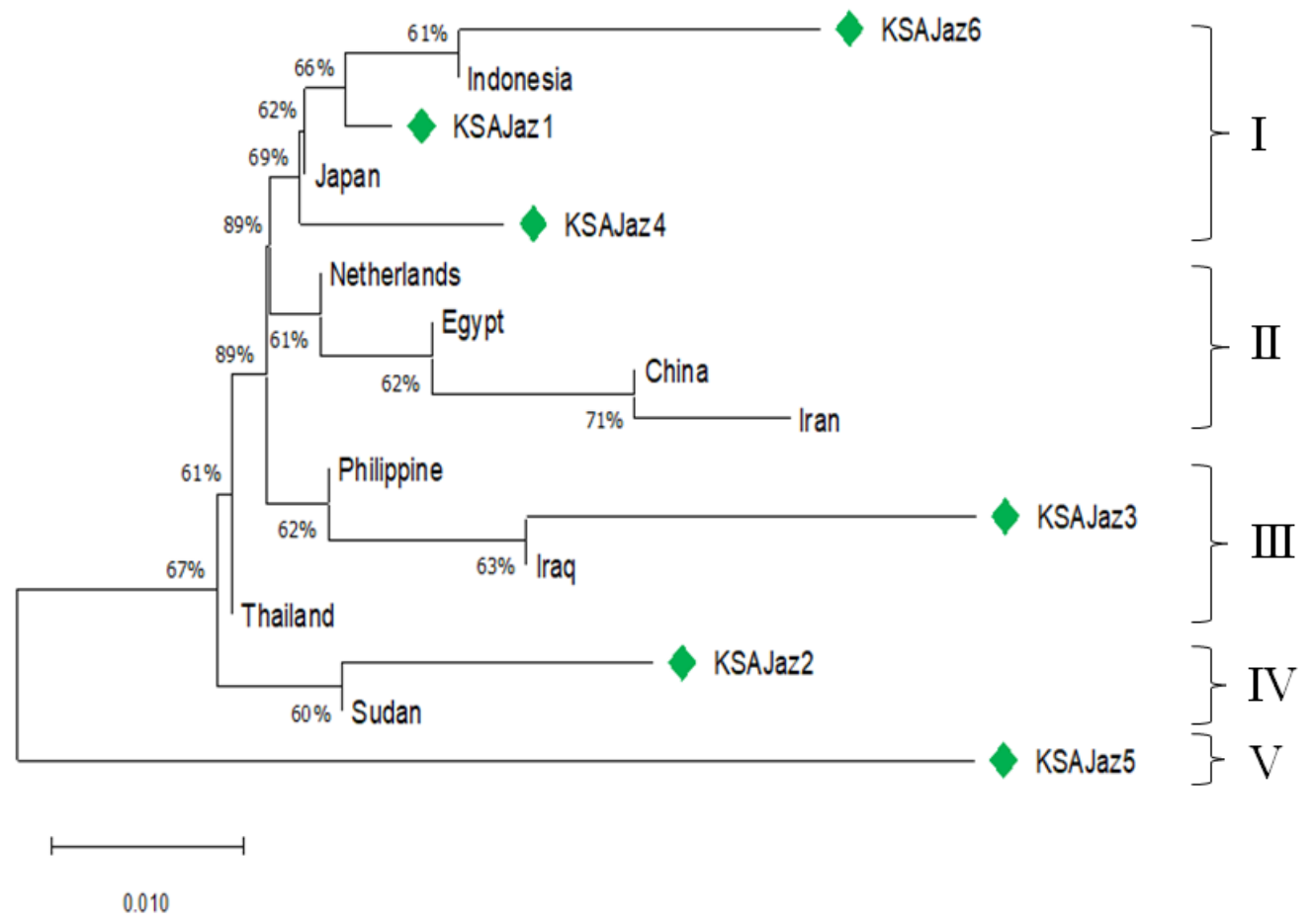


Table.1 Pairs of primer were used for T. evansi identification and quantitation.

\begin{tabular}{|c|c|c|c|c|}
\hline $\begin{array}{l}\text { Target } \\
\text { gene }\end{array}$ & Primers & Primer Sequences 53 & $\begin{array}{c}\text { Ampliconlength } \\
\text { bp }\end{array}$ & Source \\
\hline \multirow{2}{*}{ T. evansi ITS1 } & ITS1 CF/BR FB & CCGGAAGTTCACCGATATTG & \multirow{2}{*}{$480 \mathrm{bp}$} & \multirow{2}{*}{$(22)$} \\
\hline & ITS1 CF/BR RP & TGCTGC GTTCTTCAACGAA & & \\
\hline \multirow[t]{2}{*}{ RoTat 1.VSG } & TeRoTat920 F & CTGAAGAGGTTGGAAATGGAGAAG & \multirow[t]{2}{*}{$151 \mathrm{bp}$} & \multirow[t]{2}{*}{ (23) } \\
\hline & TeRoTat1070 R & GTTTCGGTGGTTCTGTTGT TG TTA & & \\
\hline
\end{tabular}

Fig.5 A part of multiple sequences alignment of ITS1 gene of Trypanosoma spp isolates from Kingdom of Saudi Arabia with known isolates (Iraq, Iran, Sudan, Egypt, Japan, China, Thailand, Netherland, Philippine and Indonesia). Identical bases are indicated by dots and predicted dissimilarity sequences are indicated by single-letter code. The sequences used were either published or present in GenBank. The comparison was made with blosum62 table, Multalin version 5.4.1 (25).


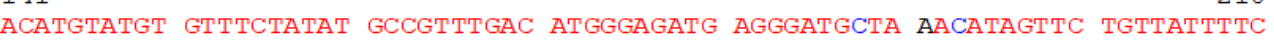

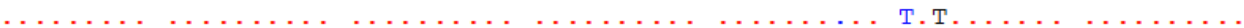


Table.2 Detection of T. evansi in camel's blood samples using microscopy and PCR based on ITS-1 and Rotat VSG genomic targets.

\begin{tabular}{|c|c|c|c|c|}
\hline \multirow{2}{*}{ Camels } & \multicolumn{2}{|c|}{ Blood film examination } & \multicolumn{2}{c|}{ PCR } \\
\cline { 2 - 5 } & Wet smear & thick smear & ITS & Rotat VSG \\
\hline Total & 454 & 454 & 100 & 19 \\
\hline positive & 14 & 16 & 19 & 7 \\
\hline Percentage \% & $3.1 \%$ & $3.5 \%$ & $19 \%$ & $36.8 \%$ \\
\hline
\end{tabular}

In the present, the prevalence rate was found to be $3.5 \%$, wherefore, we could attribute this rate to the possibility of several reasons concern with ecology of pathogen and/or diagnostic techniques, such as; i) scarcity of the rainy season of respective year is proportionally reflected on the prevalence of enzootic diseases, as Luckin (31) reported; seasonal and environmental influences will affect the population density of the insect vectors and hence the opportunities for transmission. ii) The sensitivity of PCR assays depends on the primers used and the number of repetitions of the target sequence in the trypanosome genome (32). iii) Level of specimens' preservation and the low concentration and/or purity of DNA play a vital role of unsuccessful PCR amplification. iv) Geographical area of the study as well as management and husbandry regimens of the camels under study (12). Genetic diversities of $T$. evansi in camels are reported from different parts of the world, but the genetic information is absent in dromedary camels of Saudi Arabia, thus, the current research considered to be the first study to investigate genetic novel and evolutionary origins of $T$. evansi isolates from naturally infected camels in the region and Saudi Arabia as a whole. The construction of lower detection rate of molecular technique observed in previous studies and the current one might be correlated with the sequence diversity of the RoTat1.2VSG gene isolated from camels, particularly in association with parasite long persistence in camels due to the chronic course of the disease (12). Moreover, it could be related to non-RoTat1.2VSG $T$. evansi, a variant that previously was reported only in Kenya (33-35). In this study the partial sequencing was achieved for ITS-1 rDNA, the BLAST search revealed that they corresponded to those data of $T$. evansi isolates of Iraq MH595480, Iran KX898420, Sudan LC492122, Egypt AB551922, Japan D89527, China FJ712715, Thailand AY912279, Netherland AF306775.1, Philippine HQ593646 and Indonesia MN121258 with homology average 92-99\%. Phylogenetic analysis showed an optimal tree clustering all those from known strains of $T$. evansi circulating globally and retrieved from GenBank with values at relevant nodes. The Phylogenetic trees based on ITS1 analyzed in the present study were informative to infer phylogenies and relatedness of the KSA isolates. Trypanosoma isolates were clustered into five main clades (I-V) for KSA with other known isolates, namely, clade I; KSAjaz6, Indonesian isolate, KSAjaz1, Japanese isolates and KSAJaz4 (bootstrap percentage 89\%), clade II; isolates from Iran, China, Egypt and Netherlands (bootstrap percentage 61\%), clade III; KSAjaz3, isolates from Iraq, Philippine and Thailand (bootstrap percentage 61\%), clade VI; KSAjaz2 and isolate from Sudan, (bootstrap percentage 60\%), whereas KSAjaz5 represents clade $\mathrm{V}$ in distinct separate branch (bootstrap percentage 67\%) (Fig.4).The notable variation detected in the current study may be associated with its role in antigenic variation of the surface receptor for evading 
host immune reactions (36). This may have a role in the chronic nature of the disease in the camels; and cope with the difference in the pathogenicity of parasites and its ability to infect a wide range of host species $(37,38)$. The finding of our study demonstrated phenotypic divergence of $T$. evansi which lead us to hypothesize that there are multiple origins of $T$. evansi phenotypes and thus availability of complex characteristics such as ability for mechanical transmission have evolved multiple times, as well, the other common specifications so as ability to sustained mechanical transmission outside the tsetse belt (39). Thus our result supports the genetic diversity within $T$. evansi that has been previously reported by Sarataphan(40), Khuchareontaworn(16) and Amer(12). Pourjafar (41), though the capacity of $T$. evansi for rapid adaptation to different host species and environments may probably be related to the high genetic variability of this parasite worldwide.

From the present study, the blood samples were collected from different ecozones of Jazan region, the phylogenetic analysis yielded six heterogeneous genotypes of $T$. evansi isolate, thence, we propose to conduct a further study to investigate the relationship between these isolates and circulating vectors, addition to design a molecular primer for isolates each separately and subject to different trypanocides to reveal the drug resistance if found in the respective region.

\section{References}

1. Kasim, A. A., (1984). Detection of Trypanosoma evansi in the Saudi Arabian Camel.J. College Sci., King Saud University 15,423-427.

2. Elobaid, I. N., (2016). Prevalence and Risk Factors Associated with Camel Trypanosomiasis in Jazan Region, Saudi Arabia. M. Sc. thesis. University of Khartoum, Sudan.
3. Al-Arabi M. E. M., M, Y. O., El-Shafie, E. I., Al-Harbi, Y. J. A., \& Al-Mekhlafi, H. M. (2019). Molecular detection of Trypanosoma evansi in camels (Camelus dromedarius) in southwestern Saudi Arabia. The Thai Journal of Veterinary Medicine, 49(1), 93-100.

4. Dargantes and Reid SA (2002). Trypanosoma evansi control and containment in Australasia. Trends Parasitol 18: 219-224.

5. Wilson, A. J., Schwartz, H. J., Dolan, R. and Olaho-Mukani, W. (1983). A simple Classification of Different Types of Trypanosomiasis Occurring in Four Camel Herds in a Selected Area of Kenya. Trop Med Parasitology, 34: 220-224.

6. Melville, S. E., Majiwa, P. A. O. and Tait, A. (2004). The African trypanosome genome in Maudlin, I., Holmes, P. H. and Miles, M. A. (Eds). CAB International, United Kingdom. Pg 39-57.

7. Desquesnes, M. and Dávila, A. M. R. (2002). Applications of PCR-Based Tools for Detection and Identification of Animal Trypanosomes: A Review and Perspectives. Veterinary Parasitology. 109: 213-231.

8. Stuart, K. Brun, R., Croft, S., Fairlamb, A., Gürtler, R. E., Mckerrow, J., Reed, S. and Tarleton, R. (2008). Kinetoplastids: Related Protozoan Pathogens, Different Diseases. The journal of Clinical Investigation. 118: 1301-1310.

9. Claes F, Büscher P, Touratier L, Goddeeris BM (2005). Trypanosoma equiperdum: master of disquise or historical mistake? Trends parasitol 21: 316-321.

10. Lai DH, Hashimi H, Lun ZR, Ayala FJ, Lukes J. (2008). Adaptations of Trypanosoma brucei to gradual loss of kinetoplast DNA: Trypanosoma equiperdum and Trypanosoma evansi a repetitive mutants of T. brucei. Proc. Natl. Acad. Sci. USA. 2008 Feb 12; 105 (6): 1999-2004.

11. Agbo, C. E., Majiwa, P. A. O., Claassen, E. J. H. M. and Roos, H. M. (2001). Measure of molecular diversity within the Trypanosoma brucei subspecies Trypanosoma brucei brucei and Trypanosoma brucei gambiense as revealed by genotypic characterization. 
Experimental parasitology. 99:123-131.

12. Amer S, Ryu O, Tada C, Fukuda Y, Inoue N, Nakai Y (2011). Molecular Identification and Phylogenetic Analysis of Trypanosoma Evansi from Dromedary Camels (Camelus dromedarius) in Egypt, a pilot study. Acta Trop 117:39-46.

13. Salim B, Bakheit MA, Kamau J, Nakamura I, Sugimoto C (2011). Molecular Epidemiology of Camel Trypanosomiasis Based on ITS1 rDNA and Rotat 1.2 VSG Gene in The Sudan. Parasit Vectors 4:31.

14. Sánchez E, Perrone T, Recchimuzzi G, Cardozo I, Biteau N, Aso PM, Mijares A, Baltz T, Berthier D, Balzano-Nogueira L, Gonzatti MI. (2015). Molecular Characterization and Classification of Trypanosoma Spp. Venezuelan Isolates Based on Microsatellite Markers and Kinetoplast Maxicircle Genes. Parasit Vectors.2015; 8: 536.

15. Hillis DM and Moritz C. (1990). An Overview of Applications of Molecular Systematic. Sinauer Associates, Sunderland.pp. 502-515.

16. Khuchareontaworn $\mathrm{S}$, Singhaphan $\mathrm{P}$, Viseshakul N, Chansiri K (2007) Genetic Diversity Of Trypanosoma evansi In Buffalo Based on Internal Transcribed Spacer Regions. J Vet Med Sci 69:487-493.

17. Croof I. M. N. Hamid, Imna M, Hamis S. Nyingilili, Sonia S, Darren B, Nahla O. M. Ali (2017). Phylogenetic Analysis of Trypanosoma evansi in Naturally Infected Camels from Sudan Based on Ribosomal DNA Sequences.American Journal of Microbiology and Biotechnology. Vol. 4, No. 6, 2017, pp. 75-82.

18. SCDSI. (2018). Saudi Central Department of Statistics and Information.

19. Al-Sharif, A, (1983). Geography of kingdom. 2nd part: Southwestern of Saudi Arabia, Mareekh, Riyadh (30) Al-Khalifa, M. S., Khalil, G.M., Hussein, H.S., Diab, F.M., (2008): Blood Parasites of Livestock in Certain Regions in Saudi. Saudi J. Biol. Sci. 15: 73-79.

20. Al-Sheik, A. A. (2011). Larval Habitat, Ecology, Seasonal Abundance and Vectorial
Role in Malaria Transmission of Anopheles arabiensis in Jazan Region, Saudi Arabia. Journal of the Egyptian Society of Parasitology, 41 (3): pp. 615-634.

21. Kelly, S., Schillinger, D., (1983). Improved Field Diagnostic Technique for Trypanosomiasis by use of a mini-centrifuge. Vet. Res, 113, 219.

22. Njiru Z.K, Constantine CC, Guya S, Crowther J, Kiragu JM, Thompson RC, Dávila AM, (2005): The use of ITS1 rDNA PCR in Detecting Pathogenic African Trypanosomes. Parasitol Res, 95:186-192.

23. Claes, F., Radwanska, M., Urakawa, T., Majiwa, P.A., Goddeeris, B.M., Buscher, P., (2004). Variable Surface Glycoprotein Rotat 1.2 PCR as a Specific Diagnostic Tool for The Detection Of Trypanosoma Evansi Infections. Kinetoplastid Biol. Dis. 3, 3.

24. Altschul, S.F., Madden, T.L., Schäffer, A.A., Zhang, J., Zhang, Z., Miller, W. and Lipman, D.J., (1997). Gapped BLAST and PSIBLAST: a new generation of protein database search programs. Nucleic acids research, 25(17), pp:3389-3402.

25. Corpet, F. (1988). Multiple Sequence Alignment with Hierarchical Clustering.Nucl. Acids Res., 16 (22), 10881 10890.

26. Kumar S., Stecher G., Li M., Knyaz C., and Tamura K. (2018). MEGA X: Molecular Evolutionary Genetics Analysis Across Computing Platforms. Molecular Biology and Evolution 35:1547-1549.

27. Tamura K., Nei M., and Kumar S. (2004). Prospects for Inferring Very Large Phylogenies by Using the Neighbor-Joining Method.Proceedings of the National Academy of Sciences (USA) 101:1103011035.

28. Saitou N. and Nei M. (1987). The Neighborjoining method: A new Method for Reconstructing Phylogenetic Trees. Molecular Biology and Evolution 4:406-425.

29. Felsenstein J. (1985). Confidence Limits on Phylogenies: an Approach Using the Bootstrap. Evolution 39:783-791.

30. Al-Khalifa, M. S., Khalil, G.M., Hussein, H.S., Diab, F.M., (2008): Blood Parasites of 
Livestock in Certain Regions in Saudi. Saudi J. Biol. Sci. 15: 73-79.

31. Luckins, A. G. (1996). Problems Associated with Infections Caused by Trypanosoma evansi in Asia. In: Proceedings of a Seminar on Diagnostic Techniques for Trypanosoma evansi in Indonesia. Husein. A., Davison. H. C., Luckins. A, G., Partoutomo, S. \&Thrusfield. M. V. (Eds). Research Institute for Veterinary Science. Bogor. Indonesia., PP. 10-17.

32. Pruvot, M., Kamyingkird, K., Desquesnes, M., Sarataphan, N., Jittapalapong, A. (2010). Comparison of Six Primer Sets for Detection of Trypanosoma evansi by Polymerase Chain Reaction in Rodents and Thai Livestock, Vet. Parasitol. 171: 185-193.

33. Ngaira, J.M., Njagi, E.N., Ngeranwa, J.J., Olembo, N.K., (2004). PCR amplification of RoTat1.2 VSG gene in Trypanosoma evansi isolates in Kenya. Vet. Parasitol. 120, 23-33.

34. Ngaira, J.M., Olembo, N.K., Njagi, E.N., Ngeranwa, J.J., (2005). The detection of non RoTat 1.2 Trypanosoma evansi. Exp. Parasitol. 110, 30-38.

35. Njiru ZK, Constantine CC, Masiga DK, Reid SA, Thompson RCA, Gibson WC. (2006). Characterization of Trypanosoma evansi type B. Infect Genet Evol. 6(4):292-300.

36. Borst, P., (1991). Transferring Receptor,
Antigenic Variation and the Prospect of a Trypanosome Vaccine. Trends Genet. 7, 307-309.

37. Bitter, W., Gerrits, H., Kieft, R., Borst, P., (1998). The Role of Transferrin-Receptor Variation in the Host Range of Trypanosoma brucei. Nature 391, 499-502.

38. Pays, E., Vanhollebeke, B., Vanhamme, L., Paturiaux-Hanocq, F., Nolan, D.P., PerezMorga, D., (2006). The Trypanolytic Factor of Human Serum. Nat. Rev. Microbiol. 4, 477-486.

39. Kamidi CM, Saarman NP, Dion K, Mireji PO, Ouma C, Murilla G. (2017). Multiple Evolutionary Origins of Trypanosoma evansi in Kenya.PLoSNegl Trop Dis 11(9).

40. Sarataphan N, Boonchit S, Sirivan C, Indrakamhang P (2004). Genetic Diversity of Trypanosoma evansi In Thailand Based on A Repeated DNA Coding Sequence Marker. in: Livestock Development Proceeding, the Golden Jubilee Museum of Agriculture, Pathumthani.

41. Pourjafar, M., Badiei. K., Sharifiyazdi. H., Challah. A., Naghib. $\mathrm{M}$ and Babazadeh.M. (2012).Genetic Characterization and Phylogenetic Analysis of Trypanosoma evansi in Iranian Dromedary Camels. Parasitol Res (2013) 112:899-903.

\section{How to cite this article:}

Elobaid, N. I., O. M. Daffalla, E. M. Noureldin and Abdalla, M. A. 2021. Phylogenetic Analysis of Trypanosoma evansi Isolates in Naturally Infected Camels from Kingdom of Saudi Arabia. Int.J.Curr.Microbiol.App.Sci. 10(04): 532-543. doi: https://doi.org/10.20546/ijcmas.2021.1004.052 